Volume 5

Issue 4 - Patient Self-Management

Article 9

$10-29-2018$

\title{
Conference Proceedings: Aurora Scientific Day 2018
}

Follow this and additional works at: https://aah.org/jpcrr

Part of the Behavioral Medicine Commons, Cardiology Commons, Diseases Commons, Family Medicine Commons, Geriatrics Commons, Medical Education Commons, Neurology Commons, Obstetrics and Gynecology Commons, Oncology Commons, Public Health Commons, and the Sports Medicine Commons

\section{Recommended Citation}

Conference proceedings: Aurora Scientific Day 2018. J Patient Cent Res Rev. 2018;5:317-27. doi: 10.17294/2330-0698.1661

Published quarterly by Midwest-based health system Advocate Aurora Health and indexed in PubMed Central, the Journal of Patient-Centered Research and Reviews (JPCRR) is an open access, peer-reviewed medical journal focused on disseminating scholarly works devoted to improving patient-centered care practices, health outcomes, and the patient experience. 


\section{Conference Proceedings: Aurora Scientific Day 2018}

Selected abstracts published herein were among more than 50 presented at the 44th annual Aurora Scientific Day research symposium. Held May 25 in Milwaukee, Wisconsin, Aurora Scientific Day provides a forum for original research conducted by faculty, fellows, residents, and other allied health professionals affiliated with Aurora Health Care, an integrated health system with hospitals and clinics in Wisconsin and Illinois.

\section{ORAL PRESENTATIONS}

The Effects of a Preoperative Physical Therapy Visit on Patient Outcomes Following Total Knee Arthroplasty

Kelly E. Schneider, Sheldon R. Garrison, Maharaj Singh, Jennifer L. Pogodzinski

\section{Aurora Rehabilitation Services; Aurora Research Institute}

Background: Total knee arthroplasty (TKA), or total knee replacement, is an effective surgical approach used to treat osteoarthritis and knee trauma. Its utilization has grown, and the accompanying financial impact has resulted in an equal need to advance physical therapy practice. Historically, physical therapy is provided postoperatively as the standard of care for TKA. An emerging approach is the inclusion of a preoperative rehabilitation physical therapy program (prehab) focused on functional skill training and education; however, its effectiveness remains untested with TKA.

Purpose: In the current study, we examined the impact of standardized prehab in the health care setting, using a single visit to determine if it improved discharge disposition and length of inpatient hospital stay compared to controls.

Methods: We retrospectively reviewed 1043 adult patients at a single institution who underwent TKA and received physical therapy from September 2015 through March 2017. All categorical and numeric variables were described using appropriate descriptive statistics. The two groups (prehab and control) were compared using chi-squared/Fisher's tests and t-test for independent samples. An alpha of 0.05 was used for all statistical tests, and all statistical analysis was done using SAS version 9.4 (SAS Institute Inc., Cary, NC).

Results: Preoperative physical therapy improved discharge disposition and decreased length of inpatient stay. We found a marked shift in the prehab group. On day $1,37.1 \%$ of prehab patients left inpatient care compared to $27.0 \%$ of controls; on day 2 , the two groups were nearly identical $(48.9 \%$ and $48.8 \%$, respectively); and on day 3, 14.0\% of prehab patients left compared to $24.2 \%$ of controls (overall: $\mathrm{P}=0.0008$ ). Prehab also improved discharge disposition, which was the percentage of people who either returned home or required other arrangements such as an inpatient subacute rehabilitation facility. We found $41.6 \%$ of prehab patients returned home for self-care compared to $23.2 \%$ of controls $(\mathrm{P}<0.001)$

Conclusion: These data suggest a single preoperative physical therapy visit improves patient outcomes following TKA. Our findings highlight the need to explore the greater utilization of preoperative physical therapy to improve patient outcomes and address reimbursement changes with bundled care coverage.
The Impact of the Bundled Hospital Elder Life Program ${ }^{\odot}$ (HELP and HELP at Home) on 30-Day Hospital Readmission

Michelle Simpson, Rebecca Weeks, Amy Driscoll, Jonny A. Macias Tejada, Maharaj Singh, Michael L. Malone

Center for Nursing Research and Practice; Aurora Lakeland and Burlington Hospitals; Aurora at Home; Department of Geriatrics, Aurora UW Medical Group; Aurora Research Institute

Background: Prevention and early identification of vulnerable older adults' risk for delirium and functional decline is critical to improving quality of care across the continuum. To our knowledge there are no known studies that examine the impact of a model of care that spans across health care settings (inpatient to skilled home care) on unplanned 30-day readmissions.

Purpose: To examine the impact of the adapted model of care (Bundled HELP) on 30-day unplanned hospital readmission among patients 65 and older who received inpatient hospital care and skilled home care in two rural hospitals and home care in Wisconsin.

Methods: A quantitative, comparative study design was used to compare outcomes between two groups of patients 65 and older who received inpatient care on a medical-surgical unit and were discharged to skilled home care from January 2015 to October 2017. The study sample included 766 patients: 617 control/preBundled HELP; 149 treatment/Bundled HELP. Key elements of the Bundled HELP include deployment of HELP on medicalsurgical units, use of the home-care-to-hospital-readmission risk score for 24- or 48-hour home care start of care, and continued provision of HELP protocols to home care patients at each home care visit (RNs, CNAs, PTs, OTs). Descriptive patient variables (ie, age, length of hospital stay, comorbidity) were analyzed using means or frequencies. Multivariate logistic regression was conducted to identify predictors of unplanned 30-day hospital readmission.

Results: The rate of unplanned 30-day hospital readmission after discharge to home care among the pre-Bundled HELP/control group was $28.5 \%(\mathrm{n}=176)$; among the Bundled HELP/treatment group, unplanned 30-day readmission rate was $16.7 \%(n=25)$. On multivariate analysis, Bundled HELP $(\mathrm{P}=0.02)$, hospital primary admission diagnosis of cancer (any type) $(\mathrm{P}=0.02)$, and Charlson comorbidity index $(\mathrm{P}<0.0001)$ were predictors of unplanned 30 day readmission. Patients who received Bundled HELP had a 1.72 times reduced risk of readmission compared to patients who did not receive Bundled HELP (odds ratio: $0.59,95 \%$ confidence interval: 0.36-0.95; $\mathrm{P}=0.02)$. 
Conclusion: With $20 \%$ or more of all inpatients 65 and older discharged from the hospital to skilled home care, the impact of this adapted and bundled model of care is significant. Given average length of hospital stay is 3 days, there is less time to deliver HELP protocols in the hospital setting. The findings of this study provide support for extending this model of care beyond acute care and replicating the study.

\section{Effect of a Novel Long-Acting Neutralizing Monoclonal ACTH Antibody (ALD1611) in the Neonatal Rat: Basal and Corticosterone Responses to ACTH and Hypoxic Stress}

Ashley Gehrand, Jonathan M. Phillips, Kevin Malott, Hershel Raff

\section{Endocrine Research Laboratory, Aurora St. Luke's Medical Center; Aurora Research Institute}

Background: The control of steroidogenesis in the neonatal adrenal gland is of great clinical interest. We have demonstrated that the newborn rat [postnatal day (PD) 2] exhibits a corticosterone response to hypoxia in the absence of an increase in plasma ACTH measured by radioimmunoassay, whereas the corticosterone response to exogenous $\mathrm{ACTH}$ is intact. By PD8, the corticosterone response to hypoxia is ACTH-dependent. This apparently ACTHindependent response to hypoxia in the newborn rat may be due to an increase in a bioactive, non-immunoassayable form of ACTH.

Purpose: To evaluate the ACTH-independent response to hypoxia in newborn rats using a novel, specific neutralizing antibody (ALD1611) to ACTH.

Methods: Rat pups ( $\mathrm{N}=6-14$ per group) were given ALD1611 (20 $\mathrm{mg} / \mathrm{kg}$, intraperitoneal) or vehicle on the morning of PD1, PD7, or PD14; 24 hours later (on PD2, PD8, or PD15), baseline blood samples and adrenal glands were obtained. Then, porcine ACTH [1-39] was injected (20 mcg/kg, subcutaneous) or hypoxia $\left(8 \% \mathrm{O}_{2}\right)$ was administered, and blood was sampled for ACTH and corticosterone and adrenal glands collected for quantitative polymerase chain reaction (qPCR) 60 minutes later.

Results: Treatment with ALD1611 decreased baseline corticosterone and eliminated the corticosterone response to ACTH or hypoxia in all age groups. This occurred despite the fact that hypoxia did not induce a statistically significant increase in plasma ACTH in the PD2 pups. In additional experiments, we found that the magnitude and duration of the attenuation of the adrenal response to ACTH injection or hypoxia was related to the dose of ALD1611 (0.1 $\mathrm{mg} / \mathrm{kg}$ to $20 \mathrm{mg} / \mathrm{kg}$, intraperitoneal). ALD1611 also blocked stressinduced changes in the expression of mRNAs (by qPCR) of critical adrenal steroidogenic pathway genes.

Conclusion: ALD1611 is highly effective in decreasing basal plasma corticosterone and in blocking the adrenocortical response to exogenous ACTH and hypoxic stress in the neonatal rat. We conclude that, despite the minimal increase in plasma ACTH, the adrenal response to hypoxia is ACTH-dependent at all age groups, suggesting stress-induced increases in alternate biologically active forms of ACTH and/or hypoxia-induced increase in adrenal sensitivity in the PD2 neonatal rat. ALD1611 may prove useful in attenuating ACTH-dependent adrenal steroidogenesis in vivo and eventually in treated patients with ACTH-dependent Cushing's syndrome.

\section{New-Onset Cardiomyopathy With and Without Atrial} Fibrillation

Vinay Mehta, Alexander J. Albers, Alex Baek, Maharaj Singh

\section{Aurora BayCare Medical Center; Aurora Research Institute}

Background: Atrial fibrillation (AF) is the most common arrhythmia and is often associated with cardiomyopathy (CM) and heart failure. There has been little research performed to assess whether there are any differences in patient profile, comorbidities, presenting complaints, and electrocardiographic (ECG) or laboratory characteristics and outcomes between patients who have $\mathrm{AF}$ at presentation or preceding the diagnosis of new-onset $\mathrm{CM}$ (defined as left ventricular ejection fraction $\leq 40 \%$ ) and patients who are diagnosed with new-onset CM without any preceding atrial dysrhythmias.

Purpose: To assess differences among patients with new-onset $\mathrm{CM}(\mathrm{LVEF} \leq 40 \%)$ with $\mathrm{AF}$ and without $\mathrm{AF}$.

Methods: We used a balance research design to compare patients diagnosed with new-onset $\mathrm{CM}$ with $\mathrm{AF}(\mathrm{N}=196)$ and without $\mathrm{AF}$ $(\mathrm{N}=197)$. Demographic characteristics, comorbidities, diagnosis, ECG characteristics, laboratory marker variables, and outcome data were described using appropriate descriptive statistics. The two groups of patients with and without AF were compared using chi-squared and independent t-test. An alpha of 0.05 was used for all statistical tests, and all statistical analysis was done using SAS version 9.4 (SAS Institute Inc., Cary, NC).

Results: Patients in the AF-CM group were older (73.51 vs 64.18 years; $\mathrm{P}<0.001)$ and more likely to be male $(63.3 \%$ vs $49.2 \%$; $\mathrm{P}=0.005)$, of Caucasian ethnicity $(96.9 \%$ vs $76.7 \% ; \mathrm{P}<0.001)$, and have a history of hypertension $(70.4 \%$ vs $53.3 \% ; \mathrm{P}=0.0005)$ or coronary artery disease $(33.7 \%$ vs $20.8 \% ; \mathrm{P}=0.0042)$. They were more likely to be diagnosed as an outpatient $(35.7 \%$ vs $10.2 \% ; \mathrm{P}<0.0001$ ); if presenting to the hospital, they were much more likely to have congestive heart failure as their presenting complaint $(61.1 \%$ vs $38.4 \% ; \mathrm{P}<0.0001)$. They also were more likely to have low voltage on their presenting ECG compared to the control group ( $39 \%$ vs $27.6 \% ; \mathrm{P}=0.0003)$. Troponin ( 0.91 vs 14.27; $\mathrm{P}=0.0067)$ and brain natriuretic peptide ( 771.3 vs 1015.6 ; $\mathrm{P}=0.021$ ) values were significantly lower in the $\mathrm{AF}$ group. Patients in the AF-CM group showed an increased risk of death: $30.1 \%$ vs $20.8 \%(\mathrm{P}=0.0345)$.

Conclusion: Patients with atrial fibrillation and new-onset cardiomyopathy differ from patients without atrial fibrillation in demographic characteristics, comorbidities, venue of diagnosis, presenting complaints, ECG characteristics, laboratory markers, and prognosis. This may have implications for diagnosis, work-up, and treatment of these patients.

\section{Validation of Stroke Network of Wisconsin (SNOW) Scale at Aurora Health Care}

Kessarin Panichpisal, Adil Chohan, Paul Vilar, Maharaj Singh, Reji Babygirija, Mary L. Hook, Sharon Matyas, Rehan Sajjad, Thomas Wolfe, Richard A. Rovin

Aurora Neuroscience Innovation Institute; Marian University College of Osteopathic Medicine; Aurora St. Luke's Medical Center; Aurora Research Institute; Aurora Sinai Medical Center 
Background: The Stroke Network of Wisconsin (SNOW) scale, previously called the Pomona scale, was developed to predict large-vessel occlusions (LVO) in patients with acute ischemic stroke. The original study showed a high accuracy of this scale.

Purpose: We sought to externally validate the SNOW scale in an independent cohort.

Methods: The SNOW scale includes 3 items: gaze deviation, expressive aphasia, and neglect. The SNOW scale is positive if any one of these items is present. We retrospectively reviewed a large cohort of all acute stroke patients who presented within 24 hours after onset at Aurora Health Care (14 hospitals) from January 2015 to December 2016. We calculated SNOW scale, the Vision Aphasia and Neglect (VAN) scale, the Cincinnati Prehospital Stroke Severity Scale (CPSSS), the Los Angeles Motor Scale (LAMS), and the Prehospital Acute Stroke Severity (PASS) scale for all patients. The predictive performance of all scales and several National Institute of Health Stroke Scale (NIHSS) cutoffs $\geq 6$ were determined and compared. LVO was defined by total occlusions involving the intracranial internal carotid artery, middle cerebral artery (M1), or basilar arteries.

Results: Among 2183 acute ischemic stroke patients, 1381 had vascular imaging and were included in the analysis. LVO was detected in 169 (12\%). A positive SNOW scale had comparable accuracy to predict LVO as the CPSS and an NIHSS $\geq 6$. With area under the receiver operating characteristics curve of 0.78 , a positive SNOW scale had higher accuracy than VAN $(0.67, \mathrm{P}<0.001)$, LAMS $\geq 4(0.62, \mathrm{P}<0.001)$, and PASS $\geq 2(0.69, \mathrm{P}<0.001)$. A positive SNOW scale had sensitivity of 0.80 , specificity of 0.76 to predict $\mathrm{LVO}$, positive predictive value of 0.31 , and negative predictive value of 0.96 for the detection of $L V O$ versus CPSS $\geq 2$ of $0.64,0.87,0.41$, and 0.95 , respectively.

Conclusion: In our large stroke network cohort, the SNOW scale has promising sensitivity, specificity, and accuracy to predict LVO. Future prospective studies in both prehospital and emergency room settings are warranted.

\section{Hot Spotting Medically Complex At-Risk Patients in an Urban Primary Care Residency Clinic}

Glenda Sundberg, Chris Peters, Catherine de Grandville, Natalie Sorenson, Rinal Patel, Melissa Grube

Aurora St. Luke's Family Practice Center; Department of Family Medicine, Aurora Health Care; Aurora Family Services

Background: In the United States, 5\% of patients incur $50 \%$ of health care costs. Hot spotting, a collaborative care approach, may improve patient outcomes and decrease health care costs among those considered to be super-users. Aurora Health Care may benefit from this new and innovative approach to care.

Purpose: The purpose of this pilot project was to create a more formalized plan for managing the most complex patients at Aurora St. Luke's Family Practice Center (Milwaukee, WI).

Methods: During 2017, 20 medically at-risk patients $\geq 18$ years of age were identified and tracked. Each patient was asked to voluntarily participate and verbally consented. Brief patient/family and caregiver surveys were conducted pre- and post-home visit. Following identification of our cohort, an interdisciplinary team meeting was conducted quarterly to discuss patients and to initiate and update care plans. The diverse medical team included a faculty physician, resident(s), clinic social worker, nurse practitioner, clinical pharmacist, registered nurse, and any other available learners. Through these meetings, we attempted to analyze and address each patient's social situation and barriers. There was at least one home visit conducted for each patient and, overall, more frequent contact with the patients and caregivers. Additionally, preand postproject hospital admissions, clinic access, and emergency department (ED) utilization was tracked.

Results: Overall, the patient population had a mean age of 62.4 years and was predominately female $(70 \%)$. The average Charlson score for patients was 6.2 (median: 6). (A score of 6 equates to a 10 -year mortality risk of $98 \%$.) Analysis of characteristics of the 20 patients revealed $80 \%$ prevalence of mental illness and communication problems; $70 \%$ were overmedicated. Comparing the 6-month periods before and after initiating the pilot, total ED visits decreased $20.0 \%$ (95 vs 76) and total admissions decreased $35.5 \%$ (31 vs 20 ). Surveys determined that patients, clinic staff, and physicians were very satisfied with the intervention. Overall, $94 \%$ of provider respondents felt more patients would benefit from being involved.

Conclusion: This well-received collaborative pilot substantially reduced hospital utilization in high-risk clinic patients. We believe this concept has large potential to provide further future benefit and may be replicated elsewhere in the system. In 2018, our pilot efforts will be extended to two residency sites for further evaluation.

\section{Differences in Metabolic Profile Between Right and Left Atria of Patients With Atrial Fibrillation}

Larisa Emelyanova, Steven M. Komas, Susan Olet, Sean Ryan, Catherine Warner, Farhan Rizvi, Gracious R. Ross, David C. Kress, Daniel P. O'Hair, Francis X. Downey

Center for Integrative Research on Cardiovascular Aging, Aurora Research Institute; Department of Biophysics, Medical College of Wisconsin; Cardiovascular and Thoracic Surgery, Aurora Medical Group

Background: Several studies have demonstrated genomic, morphological, and electrophysiological differences between the right atrium and left atrium, suggesting that dissimilar mechanisms may contribute to the development and progression of atrial fibrillation (AF). Therefore, differences in metabolic response to $\mathrm{AF}$ between atria are foreseeable. Given the complexity of AF development and progression, understanding AF-associated changes in metabolites in both atria will help in better clinical management of AF.

Purpose: To compare potential changes in metabolites in the right atrial (RAA) and left atrial (LAA) appendage tissue from patients with $(\mathrm{AF}+$ ) and without (non-AF) history of $\mathrm{AF}$.

Methods: RAA and LAA tissue from AF+ $(n=20)$ and non-AF $(n=20)$ patients undergoing elective open heart surgery at Aurora St. Luke's Medical Center (Milwaukee, WI) was collected. The tissue was snap-frozen in liquid nitrogen and stored at $-80^{\circ} \mathrm{C}$. Metabolites were profiled in frozen tissue using high-performance liquid chromatography coupled to tandem mass spectrometry (LCMS). Comparison between groups was done using the 2 sample t-test and Wilcoxon rank-sum test, with $5 \%$ level of significance. The study was approved by the local institutional review board.

Results: A total of 24 metabolites related to glycolysis and tricarboxylic acid cycle (TCA) were identified. The most significant AF-associated changes in metabolites were observed in RAA compared to LAA tissue. In $\mathrm{AF}+$ patients, glycolysis metabolites' level of glucose-6-phosphate $(\mathrm{P}=0.03)$ and 
phosphoenolpyruvate $(\mathrm{P}=0.03)$ was significantly reduced in RAA, while 2-phosphoglycerate $(\mathrm{P}=0.02)$ level was reduced in LAA. AF+ was associated with a significant decrease in TCA metabolites, including NAD $+(\mathrm{P}=0.03), \mathrm{GDP}(\mathrm{P}=0.05)$, and citrate $(\mathrm{P}=0.03)$, in RAA without considerable changes of metabolites in LAA. In addition, there was AF-associated decrease in the total pool of adenine nucleotides (ie, AMP + ADP + ATP, P=0.02) and glutathione level $(\mathrm{P}=0.03)$ in RAA when compared to LAA.

Conclusion: Atrial fibrillation is associated with different metabolic profiles between right and left atria. This may reflect different metabolic mechanisms underlying right and left atrial involvement in AF patients and may help to reveal potential chamber-specific biomarkers and targets for better preventive strategies and development of novel cardioprotective interventions.

\section{POSTER PRESENTATIONS}

\section{Isolation of Cryptococcus-Like Yeast From Natural Environments}

\section{Sarah L. Ward, Brian Hoeynck, Dennis J. Baumgardner}

Department of Family Medicine, Aurora UW Medical Group; Aurora Research Institute; Center for Urban Population Health

Background: Cryptococcus (C. gatti and C. neoformans) causes meningitis and pneumonia in immunocompromised and immunocompetent hosts, and several cases are diagnosed at Aurora Health Care each year. Cryptococcus is infrequently isolated from the environment, despite being the presumed infection source, with no known isolations in Wisconsin since 1964. C. gatti-endemic areas are expanding worldwide, and it is commonly isolated from tree hollows.

Purpose: To isolate pathogenic Cryptococcus from Wisconsin natural sites.

Methods: Samples were obtained from tree structures and natural and built surfaces in northern and southern Wisconsin (103 samples) and northeast Ohio (8) from April 2017 to December 2017. Cotton (38 samples) and liquid Amies elution (73) swabs were used to collect material for incubation at $35^{\circ} \mathrm{C}$ (after first 18 samples were at $20^{\circ} \mathrm{C}$ ) on Staib (birdseed) agar. Suspicious colonies were further incubated on Sabouraud dextrose and brain-heart infusion agar at $20^{\circ} \mathrm{C}$ and $37^{\circ} \mathrm{C}$, respectively, and on urea agar. Colonies were further examined microscopically with India ink.

Results: Use of liquid Amies elution swabs and isolation at $35^{\circ} \mathrm{C}$ reduced background mold growth. Of 111 samples, 2 isolates of Cryptococcus-like yeast were identified from the same weeping willow tree in Greendale, Wisconsin. These tan isolates on Staib agar were very similar in appearance, grew at $37^{\circ} \mathrm{C}$, and were urease-positive, but had thin, rather than broad, capsules. One isolate tested at ACL Laboratories (Milwaukee, WI) using matrix-assisted laser desorption/ionization (MALDI) technology did not match with any database organism, which, combined with phenotypic findings, suggests that these isolates likely represent nonpathogenic environmental Cryptococcus species. No putative pathogenic Cryptococcus was isolated from these samples, consistent with the $0-10 \%$ isolation success reported in the literature.

Conclusion: Isolation of these Cryptococcus-like yeasts suggests that further isolation attempts with this technique may result in isolation of pathogenic Cryptococcus strains from the environment in Wisconsin.
Characteristics of Patients in the Specialty Access for Uninsured Program

Sally Smaida, Widalys Calderon Torres, Jessica J.F. Kram, Michelle Tong, Stephen J. Ohly, Dennis J. Baumgardner

Aurora Walker's Point Community Clinic; Department of Family Medicine, Aurora UW Medical Group; Center for Urban Population Health

Background: The Specialty Access for Uninsured Program (SAUP) is a Milwaukee County health system collaborative in which safetynet primary care clinics are paired with hospital/health systems. The clinics provide primary care services, while the hospitals provide a network of specialists. All specialty services are "covered" under SAUP at no cost.

Purpose: To examine the clinical, geodemographic, and referral pattern features of our SAUP patients and their journey to specialized care.

Methods: We prospectively identified and retrospectively reviewed patients $\geq 18$ years of age residing in Milwaukee County that were enrolled in SAUP during 2017. To be eligible for SAUP (ie, managed care) patients must be established patients of Aurora Walker's Point Community Clinic (Milwaukee, WI), 200\% at or below the Federal Poverty Level, and unable to secure public or private insurance. Several variables of interest were retrospectively collected after initial SAUP enrollment. Descriptive statistics were used to describe the overall characteristics of our 2017 SAUP cohort. Regression was used to explore predictors of time from referral to specialty visit.

Results: Of the 99 patients enrolled in SAUP, 52.5\% were female. The patient population had a mean age 46.5 years and body mass index of $30.9 \mathrm{~kg} / \mathrm{m}^{2}$. Patients were predominately Hispanic (98.0\%), Spanish-speaking $(93.9 \%)$, had contact with primary care prior to enrollment (84.4\%), and resided in 2 ZIP codes within Milwaukee County (85.9\%). At the time of SAUP enrollment, patients primarily had a clinical history of hypertension $(21.2 \%)$ and diabetes $(23.2 \%)$. Overall, SAUP-enrolled patients were generally well, with $76.5 \%$ of patients having a modified Charlson comorbidity index score of zero. Of the patients enrolled in SAUP, $90.9 \%$ followed through with the specialty visit to date. Top specialty services to which patients were referred included obstetrics (13.1\%), colonoscopy/ colorectal surgery $(12.1 \%)$, and ophthalmology $(11.1 \%)$. The mean time between enrollment and specialty service visit was 30.1 days (median: 24.5 days), and no predictive variables were identified. Following the specialty services visit, $42.2 \%$ had contact with a primary care provider to date.

Conclusion: Patients in a managed-care specialty access program coordinated through our Milwaukee free clinics are relatively young and healthy, with follow-through percentages and wait times for specialty care at or better than national averages. Further research, including cost outcomes, is warranted.

Hospital Elder Life Program: A Retrospective Quality Improvement Project of a Delirium Prevention Program

Jonny A. Macias Tejada, Marianne Klumph, Regina Manansala, Jessica J.F. Kram, Candice R. LeGros, Ariba Khan

Hospital Elder Life Program, Aurora St. Luke's Medical Center; Aurora UW Medical Group; Center for Urban Population Health; Aurora Research Institute; Center for Senior Health and Longevity, Aurora Sinai Medical Center 
Background: Delirium is an acute change in mental status commonly seen in hospitalized older adults. Although the occurrence of delirium is serious and is associated with adverse outcomes, patients with delirium may be prescribed antipsychotics/ benzodiazepines, which may lead to potential side effects and other complications. The Hospital Elder Life Program (HELP) is a national evidence-based program designed to prevent delirium and functional decline. Research demonstrates that HELP decreases the onset and/or length of delirium episodes, functional decline in hospitalized older adults, and readmission rates, promotes nonpharmacological interventions, and is a cost-effective method for improving outcomes.

Purpose: To evaluate 30-day readmission percentage and assess the prevalence of newly prescribed antipsychotic/benzodiazepine medication prior to discharge among HELP-enrolled patients at a large medical center.

Methods: We conducted a retrospective study of patients $\geq 65$ years of age who were admitted and enrolled into 1 of the 8 HELP units in 2016 or 2017. Patient characteristics were described using basic descriptive statistics. Odds ratios were calculated as the odds of having antipsychotics/benzodiazepines prescribed at admission versus those who were not. Chi-squared tests were performed to detect statistical differences among nominal variables. A P-value less than 0.05 was deemed significant.

Results: 1400 patients (mean age: 80; range: 65-100) were enrolled into HELP. Overall, 25\% $(n=355)$ of patients were recorded to have been readmitted within 30 days of initial discharge from a HELP unit. This number was not significantly associated with antipsychotic/benzodiazepine prescription at admission $(\mathrm{P}=0.8575)$. Yet, when examining sex differences, females patients were more likely to receive a new antipsychotic/benzodiazepine prescription at admission $(\mathrm{P}<0.0001)$. Similarly, having a mental illness diagnosis at the time of admission made one more likely to have antipsychotics/benzodiazepines prescribed $(\mathrm{P}<0.0001)$.

Conclusion: One-quarter of patients in HELP were readmitted within 30 days of discharge. Moreover, patients enrolled in HELP with a mental illness diagnosis prior to admission were more likely to receive antipsychotic/benzodiazepine prescription at admission. Further studies could assist defining steps to decrease high-risk medications prescribed to HELP-enrolled patients with a history of mental illness.

\section{Improving the Quality of Life of Patients With Chronic Pain: A Pilot Study}

Betty Amuzu, Jessica J.F. Kram, Niraj Nijhawan

Department of Obstetrics and Gynecology, Aurora UW Medical Group; Center for Urban Population Health; Department of Anesthesiology, Aurora Medical Group

Background: Mind and body tools, such as exercise and meditation, can impact pain-reducing brain areas and lessen the pain experience for patients with chronic pain.

Purpose: To see if introduction of mind/body tools has measureable improvement on quality of life for patients with chronic pain.

Methods: As previously reported, we conducted a pilot study on adult women with stable chronic pain who were resistant to conventional therapies. Our intervention began in June 2015 and consisted of an initial 8-hour session. Following the initial session, meetings were 1.5 hours long weekly for 8 weeks, followed by biweekly meetings for 8 weeks, then monthly.
Mind/body tools (ie, deep meditation, breath work, etc) were systematically taught and reinforced during meetings. World Health Organization Quality of Life-BREF (WHOQOL-BREF) surveys were completed periodically throughout the pilot study. The WHOQOL-BREF has subcategories for overall quality of life, physical health, psychological health, social relationships, and environmental health. Paired t-tests were used to compare patients at baseline and 24 months post-mind/body tool education.

Results: As previously reported, women $(\mathrm{N}=5)$ were of mean age 43.2 years and body mass index of $35.8 \mathrm{~kg} / \mathrm{m}^{2}$. Mean longacting narcotic (LAN) was 260 morphine equivalents at baseline and now 68 morphine equivalents at 24 month assessments, respectively. Patients did not significantly decrease use of LAN. Only 4 patients completed the WHOQOL-BREF survey at 24 months. There was no statistical difference in patients' overall quality of life, physical health, psychological health, social relations, and environmental health from baseline to conclusion of the study. Even so, ongoing verbal feedback universally praised the program and may be reflected in the changes we noticed in overall QOL within the first 3 months of the program. Ultimately, it is also notable that for most, the greatest benefits were seen at 3 and 6 months while the program was still ongoing. Most benefits were not maintained beyond active participation in the program.

Conclusion: Given that this was a pilot study with a small sample size, there were no significant findings or even notable long-term trends in measurable outcomes. While narcotic use decreased, patient use may have reduced because of the current changes in regulations and guidelines for narcotic use, perhaps in combination with this program. Further study is warranted.

\section{Identification of New Therapeutic Drugs for Triple- Negative Breast Cancer}

\section{Paul Mintz, Judy A. Tjoe}

TORQUE, Aurora Research Institute; Surgical Oncology, Aurora Cancer Care

Background: The development of cancer is a multistep process of sequential genetic alterations in oncogenes and tumor-suppressor genes, making it extremely challenging to find a cure for cancer. In the case of breast cancer, patients diagnosed with triple-negative breast cancer $(15 \%-20 \%)$ have the worst survival outcome and no new treatment options. It is characterized by having no or low expression level of three genes, estrogen receptor (ER), progesterone receptor (PR), and human epidermal growth factor receptor type 2 (HER2), which makes it more difficult to develop therapeutic agents. A new targeting approach is greatly needed to identify novel drug targets for triple-negative breast cancer.

Purpose: We have taken a drug-targeting approach to identify new drug targets for triple-negative breast cancer. We propose to test Food and Drug Administration (FDA)-approved drugs on patientderived triple-negative breast cancer primary cells generated from patient tissue.

Methods: Residual tissue samples from triple-negative breast cancer surgery were processed and the dissociated cells were grown in a specific tissue-culture medium containing supplements. A triple-negative primary cell line was authenticated by an independent company to demonstrate that it was a true cell line (DNA fingerprinting). A panel of FDA-approved drugs was tested on the primary cell line to identify new therapeutic drugs. 
Molecular and cellular assays, including cell proliferation and cytotoxicity, were used to determine the effect of the drugs on the cells. Specific cell signaling pathways were investigated to discover the mechanism of action.

Results: We have successfully established a triple-negative breast cancer patient-derived primary cell line, the first at Aurora Health Care. The triple-negative cells are negative for the three known receptors (ER, PR, HER2) but positive for cytokeratin 5 and 8/18 by immunocytochemical staining. We have identified several potential FDA-approved drugs that inhibit triple-negative cells from growing. Selected drugs are being further investigated for their mechanism of action.

Conclusion: To address the limited treatment options for patients diagnosed with triple-negative breast cancer, we have taken a drugtargeting strategy. Repurposing FDA-approved drugs for triplenegative breast cancer could be a powerful approach to discovering new therapeutics to improve patient care.

\section{'Difficult Teaching Case' Conference Call Series - A Faculty Development Strategy}

Deborah Simpson, John R. Brill, Lonika Sood, Andy Anderson, Jennifer Hartlaub, Anne Getzin, Kjersti E. Knox, Carla J. Kelly, Will Lehmann, Cynthia Haq

Departments of Family Medicine, Academic Affairs, Population Health, and Obstetrics and Gynecology, Aurora UW Medical Group; Department of Medicine, Aurora BayCare Medical Center; University of California, Irvine

Background: Teaching requires a unique knowledge and skill set, from competency-based assessment to digital fluency and teaching of interprofessional teams. However, barriers to faculty development such as lack of time to prepare, incentives, and isolation/lack of support for one's identity as a clinician teacher are well enumerated, as are the elements of successful programs.

Purpose: To implement an ongoing faculty development case conference call series that expands participants' teaching strategies, increases their confidence as educators, and strengthens their connections to other teachers.

Methods: Case discussion is signature pedagogy in medical education as it makes the reasoning underlying one's decisions as a clinician or teacher visible and promotes deeper learning. Using a teaching-focused case methodology, we implemented a monthly 45-minute teaching case conference call series. Participants receive a one-screen email precis of the case 1-2 days in advance and then dial into a conference call. The case is sequentially reviewed with conference participants asking questions/explaining how they may frame the "assessment" and "plan." The conference ends with key teaching pearls/takehome points, with follow-up readings/resources distributed postconference and a brief evaluation.

Results: Cases have ranged from clinical teaching (a learner who "never got feedback") and small-group teaching (a learner who was offended by a teacher's analogy), to unprofessionalism (disruptive/argumentative learners), to teaching the "rock star" residents. Average number of participants was 7, with an upper limit of $11 ; 18$ different physicians and 8 different nurse practitioners have attended at least 1 of the 10 sessions. Evaluations reveal that all participants agree that the case scenario was relevant/ important, expanded teaching strategies, and connected them to others who value teaching. Typical comments include "these sessions are extremely valuable and will improve our learning culture..." and "it's a relief that I'm not the only one to have had this happen...."

Conclusion: Clinical teachers highly value the difficult teaching case conference call series. The approach is easily transferable to other organizations, requiring no advance participant preparation, and uses telephone access to offer a "safe" place to explore and learn from colleagues' difficult teaching situations and affirm their roles and value as teachers.

\section{Evaluating Well-Being in OB/GYN Residents and Faculty}

Erika Copperman, Naomi Light, Carla J. Kelly, Deborah Simpson

Department of Obstetrics and Gynecology, Aurora Sinai Medical Center; Departments of Obstetrics and Gynecology, Family Medicine, and Academic Affairs, Aurora UW Medical Group

Background: Between $22 \%$ and $60 \%$ of practicing physicians are reported to have experienced burnout. $\mathrm{OB} / \mathrm{GYN}$ resident burnout has been reported at $90 \%$. Duty-hour limitations were implemented for patient safety and have been associated with some increase in overall resident quality of life, but also potential sacrifices in resident education and patient care. Contributors to burnout and drivers of engagement include workload and job demands, control and flexibility, and poor work-life integration.

Purpose: To evaluate the impact associated with implementing limited weekend rounding, redistribution of postpartum rounding on weekdays, and introduction of quarterly wellness mornings on overall resident and faculty well-being.

Methods: Effective July 2, 2017, two workload protocols were changed. Weekend rounding protocols continue to have residents round on all antepartum and gynecology patients at the end of each 24-hour shift, but now faculty complete all postpartum rounding. Weekday postpartum rounding was redistributed, decreasing the number of patients per junior resident from $>10$ patients to a maximum of 6-7 patients per resident. Quarterly wellness mornings began in September 2017, using protected education time for faculty and resident physicians. A brief 3-item well-being check-in card (WBCIC) was developed and used to obtain participants' quarterly ratings regarding the adequacy of time for wellness, level of meaning in their work, and comments. Debriefings during resident-required curriculum time provided perspective on impact of reduced rounding protocols.

Results: Mean WBCIC scores revealed time spent on personal well-being at baseline and 7 months postimplementation was 1.6 and 2.1 for residents, respectively, and 2.4 and 2.4 for faculty (Scale: 1=pitiful to $4=$ =xcellent). The item "Work I do is meaningful to me" scored 4.9 and 4.1 for residents pre- and postintervention and 5.5 and 6.3 for faculty (Scale: 1=strongly disagree to $7=$ strongly agree).

Conclusion: After changes in rounding, residents' personal well-being improved but meaning in work declined. In contrast, faculty's personal well-being remained constant and meaning in work increased. Additional well-being interventions focused on residents' meaning in work will be explored. 


\section{Initiation of a Hyperthermic Intraperitoneal Chemotherapy Program at Aurora Health Care}

Aaron H. Chevinsky, Wesley Papenfuss, Federico A. Sanchez, James L. Weese

Surgical Oncology and Medical Oncology, Aurora St. Luke's Medical Center; Aurora Cancer Care

Background: The use of hyperthermic intraperitoneal chemotherapy (HIPEC) along with radical debulking has been the standard treatment for peritoneal-based malignancies, including appendiceal cancer (APPX), primary peritoneal cancer (PPC), peritoneal mesothelioma, and peritoneal spread from colorectal (CRC), gastric (GA), and gynecologic malignancies. At Aurora Health Care, a HIPEC program was initiated by our multidisciplinary gastrointestinal cancer group.

Purpose: To review the initiation and implementation of a HIPEC program started at Aurora.

Methods: Our protocol involves preoperative computed tomography scan, colonoscopy, upper endoscopy, and presentation at a multidisciplinary meeting. All patients received preoperative chemotherapy. All patients had diagnostic laparoscopy to determine resectability prior to debulking (same day). Laparotomy with complete debulking and resection of visually involved tissue was then performed. After temporary abdominal wall closure, HIPEC was performed for 90 minutes, chemotherapy was flushed and drained, and anastomoses were created. Patients were kept on chemotherapy precautions in the intensive care unit for a minimum of 48 hours.

Results: From October 2016 to June 2017, a total of 12 patients 28-76 years of age were referred for HIPEC; 10 patients had HIPEC (90 minutes at $\left.42^{\circ} \mathrm{C}\right)$ utilizing mitomycin $\mathrm{C}(30 \mathrm{mg}$ at time 0 and $10 \mathrm{mg}$ at 60 minutes) after complete debulking of their tumor. Diagnoses included APPX (4), PPC (2), ovarian cancer (2) and CRC (2). Two patients did not undergo HIPEC due to extensive disease (GA and APPX). Of the 10 patients who had HIPEC, 9 had prior surgeries (mean: 1.8, range: 1-4). Peritoneal carcinomatosis index score ranged from 4 to 19. Resections included colon (7), spleen (3), diaphragm (3), small bowel (2), liver (2), stomach (1), gall bladder (1), pancreas (1), abdominal wall (1) and ovary (1). No patients had anastomotic leakage. Length of stay ranged from 7 to 54 days (mean: 15.8, median: 10). All patients had complete debulking; 3 received postoperative chemotherapy and 2 (APPX and CRC) have recurred. Postoperative complications have included prolonged ileus, recurrent small bowel obstruction, and intraabdominal collections and abscesses. There were no mortalities.

Conclusion: Cytoreduction and HIPEC are feasible in a large community-based health system. Our results were favorable and, after our initial evaluation, we plan to continue our program and move forward with an institutional review board-approved study looking at tissue and blood levels of mitomycin $\mathrm{C}$ prior to, during, and after HIPEC.

\section{Variability in Sentinel Lymph Node Biopsy Retrieval for Breast Cancer at Aurora Health Care}

Aaron H. Chevinsky, Judy A. Tjoe, William L. Owens, James L. Weese

Surgical Oncology, Aurora St. Luke's Medical Center; Aurora Cancer Care; TORQUE, Aurora Research Institute; Surgery, Aurora BayCare Medical Center
Background: Axillary lymph node involvement has always been one of the most important factors in determining treatment and prognosis for breast cancer. Routine axillary lymph node dissection (ALND) was the standard treatment for breast cancer until the mid-1990s, when sentinel lymph node biopsy (SLNB) became the standard.

Purpose: We undertook an evaluation of the SLNB procedure, comparing dedicated breast surgeons (4) with general surgeons who also perform breast procedures (25) to see if there were any differences and to standardize the approach throughout Aurora Health Care.

Methods: We performed a retrospective chart review at Aurora to evaluate patients undergoing surgical treatment for breast cancer. The audit revealed that over a 6-month period from January 1, 2016, to June 30, 2016, 25 general surgeons and 4 dedicated breast surgeons performed 275 surgeries for primary operable breast cancer (stages I-III). There were 180 lumpectomies (LUMP) and 95 mastectomies (MAST) performed.

Results: In the 275 breast cancer operations, 253 (92\%) SLNB procedures were attempted (163 LUMP, 90 MAST). For various reasons, 13 patients in the LUMP group and 10 patients in the MAST group did not undergo SLNB and were excluded from this analysis. Nonmigration of contrast was noted in 6 patients ( 3 in LUMP group, 3 in MAST group); 4 of these subsequently had an ALND and 2 had no further axillary treatment. A mean of 2.26 and a median of 2.0 sentinel lymph nodes per patient were removed in the LUMP group. The full-time breast surgeons performed 112 SLNB operations (44\%) (range: $22-43$ operations/surgeon). The SLNB variability between groups showed the breast surgeons removed a mean of 2.37 nodes/patient for LUMP and 2.67 nodes/ patient for MAST. The general surgeons removed a mean of 2.66 nodes/patient for LUMP and 3.28 nodes/patient for MAST.

Conclusion: The following recommendations were made within the hospital system: Dual tracer imaging should be used. One sentinel lymph node is insufficient for complete evaluation, and more than 4 nodes does not improve staging. Goal is to remove all sentinel lymph nodes that are hot, blue, or palpable, with a goal of 2 to 4 nodes per patient. A complete ALND can be safely omitted in stage I-III patients having LUMP with radiation when there are only 1 to 2 sentinel lymph nodes involved. In patients $>70$ years of age who have a T1 or T2 estrogen receptor-positive tumor, SLNB can be safely omitted. SLNB after neoadjuvant therapy can be done even with an initially positive lymph node.

\section{The Aurora Caregiver Wellness Program: Weight Loss and Health Insurance Claims Cost Reductions Among the Obese Population}

\author{
George C. Mueller, Kelly Sutton
}

Population Health and System Wellness, Aurora Health Care

Background: Aurora Health Care's caregiver wellness program provides incentives and resources to encourage weight loss for obese caregivers and spouses. Quantifying the benefits of these programs to the participants and to the organization was examined, including overall shift in body mass index (BMI) and change in health care claims costs for those who participated in the program.

Purpose: To understand the impact of an incentivized weight loss wellness program and the relationship between weight loss and caregiver health insurance claims costs. 
Methods: Annual BMI screening data and health insurance claims costs were analyzed using a multiple regression model to examine overall weight loss, BMI shift, and health insurance claims costs pre- and post-weight loss program participation.

Results: Over 60 tons of excess weight loss in first 5 years (20132017). A multiple regression model shows claims costs go down $\$ 20$ for every 1 pound decrease in weight. Mean medical claims costs dropped by $\$ 3535$ for the year after participation in one specific weight loss program when compared to prior-year costs. Pharmacy claims did not show a reduction from pre- to postprogram periods. Conclusion: This research shows some support for the interventions targeting obesity in a workplace setting and the idea that weight loss results in lower health care costs.

\section{Reimagining Solidarity to Confront Infant Mortality}

\section{Alyson D. Capp}

\section{Ethics Program, Aurora Health Care}

Background: In Milwaukee, black babies die nearly three times as often as white babies, and black women experience stillbirth two and a half times more often than white women. There are many contributors to this reality. While parental responsibility is one factor, deeper, more systemic issues related to racial inequality and class privilege are also at play. Even among groups with similar socioeconomic status, racial disparities in infant mortality persist. Purpose: This project seeks to answer multiple research questions, foremost: How do socioeconomic and racial/ethnic factors relate to infant mortality in the concrete lives of black women in Milwaukee?

Methods: This research draws from ethnographic fieldwork as a type of qualitative method that puts Milwaukee women in conversation with those professionals who work to end racial disparities in infant mortality. Ethnography is a method that seeks to listen to particular persons from within their cultural milieu to better understand their values, beliefs, and practices and learn from them about matters that carry moral meaning. The type of triangulation common to ethnography, in which researchers integrate ethnographic interviews, quantitative studies, and ethical analysis, has been argued to be especially fitting to the goals of medicine. Collaborators for this project included 3 Milwaukee mothers; 5 church support group leaders, 3 of whom are nurses; 3 public health personnel; and 2 physicians.

Results: Analysis of interview recordings and transcripts uncovered 3 themes related to women and infant health: violence and stress; social hierarchy and "feeling less than;" and faith and resiliency. Women's experiences of violence and stress provide particular instances that mirror statistical connections in the literature between stress and premature birth. Stories of feeling "less than" in health care situations point to data concerning racial health disparities in care quality and outcomes. Women practice faith and resiliency amid adversity to help overcome some of these barriers. The insights of these collaborators may prove helpful in redirecting efforts to improve racial disparities in infant mortality. Conclusion: Those responsible for efforts to reducing racial disparities in infant mortality can learn important lessons from the experiences of black women in Milwaukee. Health care professionals in particular should learn from these experiences to inform how they can revise and implement strategies to reduce infant mortality.

\section{Monitoring Lead Screening Within a Milwaukee Family Medicine Residency Clinic}

Kristin E. Dement, Jessica J.F. Kram, Dennis J. Baumgardner, Bonnie Bobot, Alonzo Jalan

Department of Family Medicine, Aurora UW Medical Group; Center for Urban Population Health

Background: Lead screenings, as part of a child's preventive examinations, are offered by many Women, Infants, and Children (WIC) clinics in the Milwaukee area. Previously, the Family Care Center (FCC) at Aurora Sinai Medical Center (Milwaukee, WI) did not have access to lead screenings performed by WIC clinics and later recorded in the Wisconsin Blood Lead Registry (WBLR). Therefore, unnecessary duplicate screenings may have occurred on children seen at FCC for their preventive exams.

Purpose: To determine if children were undergoing unnecessary duplicate lead screenings at FCC.

Methods: We conducted a retrospective review of lead screenings performed at well-child exams in children 1-5 years of age at FCC from March 2017 to August 2017. We reviewed FCC patients in the WBLR, gathering additional lead screening information, noting that lead levels were often reported to nearest whole number. Screenings performed less than 6 months apart in children age 12-24 months and less than 12 months apart in children age 2-5 years were considered duplicate lead screens. Basic descriptive statistics were calculated. Categorical data were analyzed using chi-squared tests and continuous variables with 2-sample t-tests or nonparametric alternative tests. Stepwise regression and binary logistic regression was used for multivariable analysis as appropriate.

Results: After excluding 10 children with elevated blood lead levels and required repeat testing, 161 were included in our analysis. Children of mean age 1.8 years were more likely to be female (54.0\%) and African American (70.2\%). Of children with at least 1 ordered lead test, $39 \%$ were not completed; mean first lead level result was 2.4. Only $20(12.4 \%)$ had duplicate lead screenings ordered, of which $12(60.0 \%)$ were ordered inappropriately (ie, ordered as a duplicate), with $9(75.0 \%)$ being ordered by FCC. Interestingly, on univariable analysis, higher lead levels were significantly associated with male gender (3.2 vs 1.8 ; $\mathrm{P}=0.022$ ) and Asian race (4.6 vs 2.1 for all other races; $\mathrm{P}=0.046$ ). On multivariable analysis, when including age, only Asian race remained significantly associated with higher lead levels $(\mathrm{P}=0.002)$. Conclusion: Inappropriate lead tests were more commonly ordered at FCC. With access to the WBLR, we can determine if patients have had lead levels drawn at outside facilities and eliminate unnecessary duplicate tests. To further aid in decreasing the number of inappropriately ordered tests, we developed a workflow for clinic medical assistants to check blood lead screening and will conduct a 6-month postintervention analysis.

\section{Utilization of Acupuncture Services in the Emergency} Department Setting: A Quality Improvement Study

John Burns, Jessica J.F. Kram, Vashir J. Xiong, Jeanne M. Stark Casadont, Tiffany A. Mullen, Nancy Conway, Dennis J. Baumgardner

Department of Integrative Medicine, Aurora Medical Group; Department of Family Medicine, Aurora UW Medical Group; Center for Urban Population Health 
Background: Patients often present to the emergency department (ED) for pain. As opioid fatalities rise, alternative treatments in the ED are warranted for pain management. Acupuncture, a nonpharmacological treatment involving the insertion of needles into skin or tissue at specific points within the body, may help to decrease acute pain experienced by patients seeking ED services. Purpose: To assess the utilization and impact of acupuncture in the ED setting for pain management.

Methods: We conducted a prospectively designed and retrospectively reviewed quality improvement study. Patients $\geq 18$ years old who presented to the Aurora West Allis Medical Center ED (West Allis, WI) during 2017 were offered acupuncture services from an acupuncturist based on their emergency severity index score (highest severity [1] - lowest severity [5]), reason for visit, and, ultimately, their physician's recommendation. Charts were further reviewed for other demographic and visit characteristics. Demographic characteristics were described using basic summary statistics. Wilcoxon signed-rank tests were used to determine differences in pre- and postacupuncture pain, stress, anxiety, and nausea scores (ie, no pain [0] - worst pain [10]).

Results: A total of 379 patients, mean age 47.5 years and mean body mass index $30.8 \mathrm{~kg} / \mathrm{m}^{2}$, received acupuncture services. Patients were predominately female $(68.1 \%)$ and non-Hispanic white $(77.0 \%)$ and presented with an emergency severity index score of $3(68.9 \%)$ or $4(24.8 \%)$. Following enrollment, patients had $8-15$ needles placed $(86.0 \%)$ and received either 20 or 30 minutes of needle time (92.6\%). Median pre- and postacupuncture pain ( $7.0 \mathrm{vs} 3.0)$, stress ( $7.0 \mathrm{vs} 0.0)$, and anxiety ( $5.0 \mathrm{vs} 0.0)$ scores were significantly different $(\mathrm{P}<0.001)$. Differences in median pre- and postacupuncture nausea scores were not significantly different, as most patients reported no nausea preacupuncture (70.7\%). Overall, patients mean length of stay in the ED was 194 minutes, with $10.6 \%$ of patients admitted to the hospital. Only $2.1 \%$ of patients presented to the ED twice within 24 hours, and none received acupuncture services more than once. Following discharge from the $\mathrm{ED},<2 \%$ received acupuncture services within 30 days.

Conclusion: Patients admitted to the ED experienced significant decreases in pain, stress, and anxiety. It is unknown whether this decrease was due to acupuncture therapy and not the synergy between opioid and acupuncture use. Ultimately, while further study is warranted, acupuncture may be an alternative treatment to opioid use during the ED admission.

\section{Enhancing Immunization Rates at Aurora Family Medicine Clinics in Milwaukee, Wisconsin}

Zeeshan A. Yacoob, Christopher Cook, Jessica J.F. Kram, Marianne Klumph, Dennis J. Baumgardner, Marisa Stanley, Paul Hunter, Fabiana Kotovicz

University of Wisconsin School of Medicine and Public Health; Department of Family Medicine, Aurora UW Medical Group; Center for Urban Population Health

Background: In the United States, only $72.2 \%$ of children (age 19-35 months) are up-to-date on all of their immunizations. This rate is even lower for children from impoverished households (68.7\%). Lack of encouragement by providers, as well as parent perceptions of vaccine safety and efficacy, have been cited as some of the reasons for lower vaccination rates.
Purpose: Our study aimed to collect and compare patient demographic data to age appropriate and comorbidity-specific vaccination rates.

Methods: We conducted a quality improvement study at Family Practice Center (Aurora St. Luke's Medical Center, Milwaukee, WI) and Family Care Center (Aurora Sinai Medical Center, Milwaukee, WI). Patients who were seen by either a PGY-2 or PGY-3 resident provider from July 2017 to September 2017 were included. Charts were randomly selected and reviewed. Using Minitab, one-way ANOVAs were generated for multivariable data, Fisher's exact tests were used for $2 \times 2$ tables, and binary logistic regression was used for vaccine completion outcomes. A P-value less than 0.05 was used to determine statistical significance.

Results: A total of 872 patients were reviewed, of which $59.3 \%$ were female, $45.7 \%$ were African American, $11.0 \%$ were refugees, and $61.0 \%$ were insured by Medicaid. Of the comorbidities studied, the most prevalent were heart/lung disease $(36.9 \%, n=322)$, liver disease $(4.9 \%, n=43)$, and diabetes $(15.7 \%, n=137)$. Overall vaccination rates were found to be higher than national averages for children $19-35$ months old ( $85.7 \%$ [ $n=28]$ vs $72.2 \%$ nationally). Adults $\geq 65$ years of age also had higher rates of pneumococcal polysaccharide vaccine (PPSV23) immunizations (77.8\% [ $n=90]$ vs $63.6 \%$ nationally). Differing rates of PPSV23 were seen for those suffering from heart/lung disease $(47.2 \%)$, liver disease (39.5\%), and diabetes (68.6\%). Also, differing rates for hepatitis B vaccination were seen among those with liver disease $(41.9 \%)$ and diabetes $(26.6 \%)$. The following groups of patients had higher rates of missed opportunities: Medicare patients $(\mathrm{P}<0.001)$, Caucasian patients $(\mathrm{P}<0.001)$, and patients at Family Practice Center $(\mathrm{P}=0.043)$. A greater percentage of individuals were up-to-date on the adult dose of tetanus, diphtheria, and pertussis (Tdap), hepatitis $\mathrm{B}$, and human papillomavirus (HPV) vaccines at Family Practice Center versus Family Care Center.

Conclusion: Baseline immunization rates at Aurora's family medicine clinics in Milwaukee, Wisconsin, are higher than national averages. However, these rates significantly differed between clinics. Analysis of postintervention data, collected following 3 lectures given to nurses and medical assistants, will be carried out to determine the effects of the educational intervention on immunization rates.

Refugees in Milwaukee: Demographic and HealthRelated Characteristics Post-Immediate Resettlement

\section{Vy T. Dinh, Fabiana Kotovicz, Natalie Sorenson}

Department of Family Medicine, Aurora Health Care; Aurora Family Services/Aurora St. Luke's Family Practice Center

Background: Since 2002, more than 13,000 refugees have resettled in Wisconsin, with two-thirds of that population residing in the city of Milwaukee.

Purpose: To explore the demographic and health-related characteristics of a growing and dynamic refugee population in Milwaukee.

Methods: We conducted a retrospective chart review of 122 established refugee patients at two family medicine residency clinics.

Results: Of the initial 268 patients who underwent the initial refugee examination, 122 (mean age: 26 years; gender: 54.0\% male, $45.9 \%$ female) established care with a provider at one of the two studied primary care clinics at least 8 months after their 
initial screening examination. Refugees were predominantly Asian (72.1\%), primarily from the countries Myanmar (43.4\%) and Malaysia (25.4\%). Additionally, while up to $20.5 \%$ spoke more than two ethnic languages, the main languages spoken were Burmese, Rohingya, and Karen. Prevalent health topics included newly diagnosed pregnancy (29\%), latent tuberculosis $(22 \%$ [92\% completed treatment]), anemia (17\%), dyslipidemia (14\%), smoking (24\%, including both current and former smokers), betel nut use (11\%), and vitamin D deficiency $(8 \%)$. Weight issues also were prevalent, with overweight body mass index $(31.1 \%)$ more prevalent than obesity $(13.9 \%)$ or underweight body mass index (7.4\%). Prevalent health systems included musculoskeletal (23.7\%) and gastrointestinal (17.2\%). Screening rates for cervical cancer, colon cancer, and breast cancer were $57.8 \%, 58.3 \%$, and $75 \%$, respectively.

Conclusion: By identifying common health care issues and sociodemographic characteristics of our refugee patients, this study will better prepare providers for a more streamlined and equitable approach toward improving the health of this vulnerable population.

\section{Integrated Treatment of Substance Use Disorders and Trauma Experiences: The Women of Worth Program}

\section{Melissa A. Lemke, Lisa Berger \\ Center for Urban Population Health}

Background: Up to $60 \%$ of women who enter substance use disorder (SUD) treatment also have been diagnosed with posttraumatic stress disorder (PTSD), which is higher than the U.S. general population of women. Traditionally, SUD and mental health treatment have occurred in separate systems, and providers in each system tend to not be dually trained. Yet, current research has found that clients with PTSD in standard SUD treatment programs experience poorer outcomes compared to their counterparts without PTSD. Given the high prevalence of PTSD among women in SUD treatment, treating both disorders at the same time and by the same clinical team may improve outcomes.

Purpose: To examine the effectiveness of the Women of Worth program, an integrated SUD and PTSD treatment program.

Methods: The study design was single-group, and measures were administered at baseline, at treatment midpoint (12 weeks), and at treatment end (24 weeks). Adult women with a SUD in need of intensive outpatient services were eligible for enrollment. The primary measures were substance use in the past 30 days as measured by the Addiction Severity Index and psychological trauma symptom severity in the past 30 days as measured by the PTSD Checklist - Civilian Version for DSM-IV (scoring range: 17-85). Sociodemographic measures also were collected. Descriptive statistics were used to analyze data along with the Friedman test, a nonparametric statistic similar to repeated measures ANOVA, to examine client outcomes.

Results: Of the 86 women (average age: 39 years [standard deviation: 10.5]) in the Women of Worth program, most were white $(70.4 \%)$, unemployed $(84.6 \%)$, and had minor children $(61.7 \%)$. Reduction over time in the number of days of substance use (median: 2 , interquartile range $[\mathrm{IQR}]: 15 ; \chi^{2}=10.67 ; \mathrm{P}<0.01$ ) and psychological trauma symptom severity (median: 51.5, IQR: $35 ; \chi^{2}=6.65 ; \mathrm{P}<0.05$ ) was statistically significant. Median substance use at the end of treatment was 0 (IQR: 2), and the median psychological trauma symptom severity score was 28 (IQR: 30).

Conclusion: Study findings show statistical and clinical significance, with the median substance use being abstinence and the median psychological trauma symptom severity score registering below one recommended clinical cut-point of 38 at study end. When looking at the ranges of scores, and the IQR of 30 for trauma at the end of treatment, it suggests the Women of Worth program may need additional program resources to address psychological trauma among the women.

\section{HeartQoL Validation in Patients With Ischemic Heart Failure}

Sara Walczak, Susan Olet, Christina Sauld, Deborah Waller, Nasir Z. Sulemanjee

\section{Aurora Research Institute; Aurora Cardiovascular Services}

Background: Patient-reported outcome measures are increasingly used in clinical trials as performance measures to evaluate quality of care. Health-related quality of life is an example of a patientreported outcome measure. The English version of the heart diseasespecific HeartQoL health-related quality of life questionnaire has previously been validated in patients with heart disease.

Purpose: To validate the English version of the HeartQoL in patients with ischemic heart failure.

Methods: Patients with ischemic heart failure meeting clinical eligibility criteria and being treated at Aurora St. Luke's Medical Center (Milwaukee, WI) completed a sociodemographic questionnaire, the HeartQoL, the 36-Item Short Form Health Survey (SF-36, and the Hospital Anxiety and Depression Scale (HADS). The following key psychometric properties of the HeartQoL were assessed: A) factor structure; B) internal consistency reliability; C) convergent validity; and D) discriminative validity.

Results: A total of 83 patients with ischemic heart failure (mean age: $65.8 \pm 11.4$ years; female gender: $30.1 \%$; New York Heart Association (NYHA) functional class II: 44.7\%; NYHA class III: 47\%; NYHA class IV: 8.4\%) completed the questionnaires. Exploratory factor and Mokken analysis both supported the bidimensional physical and emotional factor structure of HeartQoL. Internal consistency reliability with Cronbach's $\alpha$ was excellent (global $\alpha$ : 0.92 , physical $\alpha$ : 0.91 ) or good (emotional $\alpha: 0.89$ ). Both convergent validity between similar HeartQoL and SF-36 subscales $(r \geq 0.75)$ and discriminative validity for all 18 a priori hypotheses (NYHA, shortness breath, anxiety and depression per HADS, physical activity, and SF-36 health transition) were confirmed.

Conclusion: The English HeartQoL questionnaire demonstrated satisfactory key psychometric properties of validity and reliability in this heart failure population. This study adds support for the HeartQoL as a core heart-specific health-related quality of life questionnaire in a broad group of patients with heart disease, including patients with angina, myocardial infarction, atrial fibrillation, implantable cardioverter-defibrillator, and valve surgery. 


\section{Is Spontaneous Termination of Atrial Fibrillation During Hybrid Ablation a Predictor of Clinical Outcome in Patients With Persistent Atrial Fibrillation?}

Lynn Erickson, David C. Kress, Firas Zahwe, Tadele Mengesha, David Krum, Jasbir S. Sra

Aurora Research Institute; Cardiovascular and Thoracic Surgery, Aurora Medical Group; Aurora Cardiovascular Services

Background: An epicardial/endocardial (hybrid) ablation approach has emerged as an option to treat a difficult population of patients with persistent atrial fibrillation (AF). Limited information exists on the importance of AF termination as a predictor of clinical outcome during hybrid ablation.

Purpose: This retrospective study aimed to demonstrate the significance of spontaneous AF termination during hybrid ablation of persistent $\mathrm{AF}$.

Methods: We studied results from 63 consecutive hybrid ablations in patients with no prior ablation, a mean ( \pm standard deviation) persistent AF duration of $19 \pm 16$ months and a mean follow-up of $27 \pm 13$ months. Patients received both epicardial ablation of the posterior left atrial wall using a small midline approach and a stepwise endocardial ablation (pulmonary vein isolation, ablation of complex fractionated atrial electrograms and/or linear lesions) with AF termination as the procedural endpoint. Spontaneous AF termination during the procedure was defined as conversion of $\mathrm{AF}$ to either sinus rhythm or atrial flutter (AFL), which subsequently converted to sinus rhythm during the above ablation techniques (Group 1). For patients not terminating to sinus rhythm with the above steps, electrical and/or pharmacological cardioversion was used (Group 2). Arrhythmia-free survival was defined as no arrhythmia (AFL or AF) following an initial 90-day period. To determine time to arrhythmia, ambulatory monitoring and/or electrocardiogram results during follow-up visits were collected. Arrhythmia-free survival at 12 months was determined using Kaplan-Meier estimates.

Results: There were no statistically significant differences between the two groups in baseline characteristics such as age, duration of persistent $\mathrm{AF}$, body mass index, $\mathrm{CHA}_{2} \mathrm{DS}_{2}$-VASc score, left atrial size, or left ventricular ejection fraction $(\mathrm{P}=\mathrm{ns}$ for all). There also was no significant difference in total ablation time between the two groups $(\mathrm{P}=0.1)$. Arrhythmia-free survival at 12 months for those with (Group 1) and without (Group 2) spontaneous AF termination during the procedure was $56 \%$ and $58 \%$, respectively ( $\mathrm{P}=0.7)$. AFfree survival was not significantly different between Group 1 and Group 2 (74\% vs 79\%, $\mathrm{P}=0.9$ ), nor was AFL-free survival (66\% vs $57 \%, \mathrm{P}=0.4)$.

Conclusion: Spontaneous conversion to sinus rhythm during the ablation procedure versus the need for cardioversion is not a good predictor of clinical outcome in a challenging population of patients with a long history of persistent AF who undergo the hybrid ablation procedure.
Predictors of Stillbirth Using a Large Database From the State of Wisconsin

Maharaj Singh, Ahmed Dalmar, Michelle O. Montgomery, David C. Merrill

Aurora Research Institute; Department of Obstetrics and Gynecology, Aurora Sinai Medical Center; Maternal and Fetal Medicine, Aurora Medical Group

Background: Stillbirth remains a significant problem in modern obstetrics. The exact causes for many stillbirths remain unexplained, although some risk factors have been identified.

Purpose: To identify the main risk factors for stillbirth using a large dataset, we examined incidence of stillbirth for the state of Wisconsin from 2012 through 2016.

Methods: We conducted a retrospective study using de-identified data for 323,034 pregnant women who gave birth to singleton babies in the years 2012 through 2016; 73\% of the women were white, 11\% African American and the remaining 16\% were from other races. Birth weight percentage by gestational age was calculated using 10th and 90th percentile of the baby birth weight in grams and was divided into three groups (small, appropriate, large). Risk factors for stillbirth were explored using univariate and multivariable logistic regression analysis.

Results: Overall, the prevalence of stillbirth, defined in the United States as loss of a baby after 20 weeks of pregnancy, is about 1 in 160 pregnancies $(6.3$ per 1000$)$. In the present study population from the state of Wisconsin, the rate of stillbirth was 4.1 per 1000 . Mean age \pm standard deviation for the women was $28.43 \pm 5.5$ years. The highest risk factor for stillbirth was small for gestational age (odds ratio [OR]: $15.6,95 \%$ confidence interval [CI]: $13.7-$ 17.8, $\mathrm{P}<0.001$ ), followed by diabetes (OR: 3.5, 95\% CI: 2.5-5.1; $\mathrm{P}<0.001$ ), chronic hypertension (OR: 1.5, 95\% CI: 1.1-1.9; $\mathrm{P}<0.009$ ), body mass index $>30 \mathrm{~kg} / \mathrm{m}^{2}$ (OR: $1.5,95 \%$ CI: $1.3-1.7$; $\mathrm{P}<0.001$ ), and every 10-year increase in the age of the mother (OR: 1.2, 95\% CI: 1.1-1.3; $\mathrm{P}<0.003$ ). In contrast, married women (OR: $0.8,95 \% \mathrm{CI}: 0.7-0.9 ; \mathrm{P}=0.009)$, women with higher education (OR: 0.7, 95\% CI: 0.6-0.8, $\mathrm{P}<0.001$ ), and women receiving infant care (OR: $0.5,95 \% \mathrm{CI}: 0.4-0.6$; $\mathrm{P}<0.001$ ) were less likely to have stillbirth.

Conclusion: Stillbirth prevalence in the state of Wisconsin is lower than the national average. Small for gestational age, which may be unrecognized intrauterine fetal growth restriction, was main predictor of stillbirth. Preventive strategies should focus on improving prenatal detection of fetal growth restriction, treatment of comorbid conditions such as diabetes and hypertension, and management for obesity among pregnant women. 